\title{
Oblique Pectoralis Anterior Musele:a Rare Anatomical Variation of the Pectoral Region
}

\author{
Kapil Dev, Shiva Kumar, Jaiprakash Gurawalia, Syed Althaf \\ Department of Surgical Oncology, Kidwai Memorial Institute of Oncology, Bangalore, \\ Karnataka, India.
}

Abstract:

The "oblique pectoralis anterior" an accessory muscle anterior to pectoralis major muscle originates from $4^{\text {th }}$ and $5^{\text {th }}$ costochondral junction with oblique orientation and inserts inferiorly to the anterior rectus sheath. We noticed an accessory muscle, superficial to pectoralis major with similar anatomy during mastectomy for carcinoma breast.

Key words: Axilla, Breast Neoplasms, Humans, Mastectomy, Pectoralis Muscles.

\section{Introduction}

The anatomical variations of muscles are usually encountered in the chest wall. Many kinds of accessory muscles have been distinguished in literature on cadaveric dissection. These muscles are often vestiges or developmental aberrations without having any functional role. The anterior chest wall is primarily contributed by pectoralis major and pectoralis minor.

The sternalis muscle, superficial to pectoralis major muscle with slight oblique orientation with the sternal border is present with equal sexual distribution. Many variants of sternalis have been described as biceps sternalis, sternomastalis with its common synonyms such as parasternalis, rectus sternalis and presternalis $[1,2]$. The "oblique pectoralis anterior" an accessory muscle anterior to pectoralis major muscle is reported once by Huber et al. in a female during a left mastectomy
[3]. Here, we presenting a same case of oblique pectoralis anterior in a female patient discovered during right mastectomy. The clinical importance of these accessory muscles is now cumulative as amongst clinicians as radiologists in the level of diagnosis, but rarely reported on diagnostic images before operation [4]. However, the development, morphology and functional view of these accessory muscles should be traced in detail.

\section{Case Report}

A 57 year old female was undergoing modified radical mastectomy for right breast cancer. At the time of mastectomy, we noticed an accessory muscle, superficial to pectoralis major muscle in right pectoral region. The muscle was originating from the fourth and fifth costal cartilage near to its costochondral junction with a small tendinous

\section{Corresponding Author: Dr. Kapil Dev}

Email: dr.kapil2010@gmail.com

Received: January 30, 2016 | Accepted: April 7, 2016 | Published Online: May 25, 2016

This is an Open Access article distributed under the terms of the Creative Commons Attribution License (creativecommons.org/licenses/by/3.0)

Conflict of interest: None declared | Source of funding: Nil | DOl: http://dx.doi.org/10.17659/01.2016.0056 
part. The muscle was oriented obliquely outward and downward. Inferiorly muscle was inserted over the anterior rectus sheath. The length of the muscle was $11 \mathrm{~cm}$ in length and maximum width was 5 $\mathrm{cm}$. Other muscles of ipsilateral pectoral region, including pectoralis major and minor, and latissimus dorsi and serratus anterior muscle was normal in dimensions and with respect origin and insertion were within normal limits [Fig.1].

\section{Discussion}

The frequency of the accessory muscle of the pectoral region is variable in different ethnic groups and sexual distribution, but yet to be established in literature. The identification of these muscles before the operation or cadaveric dissection is very hard due to lack of reliable clinical tests.

At the $5^{\text {th }}$ week of development, myoblasts migrates out of the last five cervical and first thoracic myotomes to derive as dorsal limb bud mass. The combination of migration, fusion and apoptosis of muscle cell precursors finally derives pectoralis muscle [5]. Either in the absence of apoptosis of designated myoblasts or myotonic hypomeres causes these accessory pectoral muscles. The numerous anatomical variations of pectoral muscles are reported in the literature. Most common anomaly of pectoral region is the complete unilateral deficiency of the pectoral major muscle defined as Poland syndrome [6]. The "atypical Poland's syndrome" is also described as bilateral hypoplasia of the clavicular head of the pectoralis major with anteriorly placed external jugular vein. The common accessory muscles of the anterior chest wall are sternalis muscle, rectus thoracis, pectoralis quartus and epicostobrachialis. Characteristically, the muscles anterior to the pectoralis major, resembled the sternalis muscle with or without additional variations. Once, it was noticed aroused from the costal region of the upper sternum anterior to the medial border of the pectoralis major as a

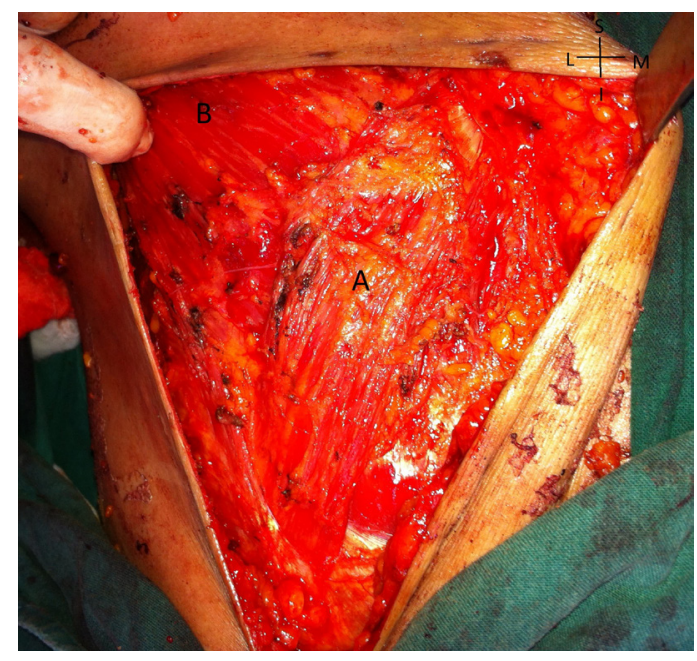

Fig.1: Intraoperative photograph of the right breast demonstrates the aberrant pectoralis accessory muscle, the oblique pectoralis anterior (A), with its fibers perpendicular to the underlying pectorals major muscle (B), Origin at $4^{\text {th }}$ costochondral junction (white arrow) and insertion at anterior rectus sheath (black arrow).

"ribbon-like strap" [7]. Another variation of the sternalis muscle arising from the external oblique aponeurosis and superiorly dividing into a " $Y$ " shape and merging with sternocleidomastoid named as "rectus thoracis bifurcalis" [8]. Morphologically, it may be a reflection of the remnant of panniculus carnosus or the derivative of pectoralis major or rectus abdominis muscles which is determined by the nerve innervation pattern. These accessory muscles usually innervated by the internal thoracic nerve or by intercostal nerves or at times both [9].

Several accessory slips of muscle in the anterior chest wall have been discovered. The "pectoralis quartus" an accessory muscle originates from $5^{\text {th }}$ costal cartilage and inserts into the coracobrachialis fascia [10]. The "epicostobrachialis" muscle arises from the lateral edge of the pectoralis major muscle and inserts into the medial epicondyle of the humerus [11]. The accessory slings of pectoralis major is an 
unilateral accessory head which inserts into the tendinous fibres of pectoralis major muscle [12]. The "chondrohumeralis" a slip arises from the pectoralis major and inserts into the biceps fascia is also reported with neurovascular complications [13]. The rectus thoracis was introduced by Vandana et al. with some variant as bifurcated insertion which was named as rectus thoracis bifurcalis. A slip situated between pectoralis major and pectoralis minor, called pectoralis minimus presented with vascular symptoms at hyperextension of the arm due to compression of the thoracoacromial vessels between pectoralis minor and minimus [14]. The "oblique pectoralis anterior" an accessory muscle anterior to pectoralis major muscle is defined by Huber et al. when he noticed during mastectomy in breast cancer patient which was used in immediate breast reconstruction [3]. In our case, we also encountered typically this accessory muscle. Although, these muscles have no functional significance except sometimes accessory muscles may enhance the optimal function of shoulder by increasing the bulk and size of the muscle.

The presence of such variant muscles may lead to the misconception in routine diagnostic modalities ultrasonography or mammography with neoplastic lesions. Post mastectomy, the un-resected accessory muscle may be confused with residual or recurrent disease in a known case of carcinoma breast. Hence, the surgeons and radiologists enforce to be familiar with these variants to avoid misdiagnosis and the use of high resolution imaging modalities as CT or MRI is obligatory to rule out the neoplastic lesions [7]. The knowledge of surgical anatomy and the planning of the reconstruction is prudent in breast and head and neck cancers which having the major burden at cancer centers. The accessory muscles have double sword inferences. Pectoralis major has enormous importance in breast cancer surgery as a surgical landmark and reconstruction of the breast and head and neck cancer surgery. Accessory muscle may cause trouble intraoperatively by disturbing the normal anatomy [15]. With the presence of accessory muscle, the pectoralis major often originates lateral to the aberrant muscle instead on the edge of the sternum. However, the understanding of the anatomical variations of anterior chest wall musculature and quickly identification intraoperative variations helps to achieve the proper dissection planes during breast and chest wall surgeries [16]. However, these rare variations should be categorized as a distinct sub-type.

\section{Conclusion}

Among the accessory muscles of anterior chest wall, this case seems to be idiosyncratic in the literature. The potential clinical significance of the present case should be debated, as the awareness of this is imperative to surgeons as well as anatomists. The understanding of these accessory muscles is important to avoid complications during surgery and to improve surgical outcomes. This case report demonstrates a need for discussing about their clinical importance.

\section{References}

1. Turner W. On the Musculus Sternalis. J Anat Physiol. 1867;1(2):246-378.

2. Anjamrooz $\mathrm{SH}$. Biceps sternalis: a $Y$ shaped muscle on the anterior chest wall. J Cardiothorac Surg. $2013 ; 8: 38$.

3. Huber KM, Boyd TG, Quillo AR, Wilhelmi BJ. Implications of anomalous pectoralis muscle in reconstructive breast surgery: The oblique pectoralis anterior. e Plasty. 2012 ; 1 2:383-389.

4. Schulman MR, Chun JK. The conjoined sternalispectoralis muscle flap in immediate tissue expander reconstruction after mastectomy. Ann Plast Surg. 2005;55(6):672-675.

5. Carlson BM. Human embryology and developmental biology. Mosby. 2004; 224225. 
6. Clarkson P. Poland's syndactyly. Guys Hosp Rep.1962;1 11:335-346.

7. Pinhal-Enfield G, Varricchio P, DeFouw DO, Vasan NS. Sternalis muscle: importance of its awareness in chest imaging and clinical significance. Int J Anat Variations. 2011 ; 4:106108.

8. Mehta V. Rectus thoracis bifurcalis: a new variant in the anterior chest wall musculature. Rom J Morphol Embryol. 2010;51(4):799-801.

9. Saeed M, Murshid KR, Rufai AA, Elsayed SE, Sadiq MS. Sternalis. An anatomic variant of chest wall musculature. Neurosciences (Riyadh). 2002;723:248-255.

10. Fabrizio PA, Hardy MA. An accessory muscle of thoracic wall. IJAV 2009;2:93-95

11. Seimon LP. Musculus Costo-epitrochlearis. SA Medical Journal. 1953;1 146-1149

12. Loukas M, South G, Louis RG Jr. A case of an anomalous pectoralis major muscle. Folia Morphol. 2006;65:100-103.

13. Lama P, Potu BK, Bhat KM. Chondrohumeralis and axillary arch of Langer a rare combination of variant muscles with unique insertion. Rom J Morphol Embryol. 2010;51(2):395-397.

14. Rai R, Ranade AV, Prabhu LV, Prakash, Rajanigandha V, Nayak SR. Unilateral pectoralis minimus muscle: a case report. Int J Morphol. 2008;26(1):27-29.

15. Dhuria R, Mehta V, Loh H, Arora J, Roy S. Suri $R$, et al. Unilateral quadripartite composition of pectoral major muscle: Anatomical description and clinical implications. Int J Morphol. 2009;27(4):1213-1216.

16. Soni S, Rath G, Suri R, Kumar H. Anomalous pectoral musculature. Anat Sci Int. 2008;83:310313. 